\title{
Prevalência de Osteoporose em Mulheres na Pós-menopausa e Associação com Fatores Clínicos e Reprodutivos
}

\author{
Prevalence of Osteoporosis in Postmenopausal Women and Association \\ with Clinical and Reproductive Factors
}

Lúcia Costa-Paiva, Ana Paula Horovitz, Alan de Oliveira Santos, Gislane Aparecida Fonsechi-Carvasan, Aarão Mendes Pinto-Neto

\begin{abstract}
RESUMO
Objetivos: avaliar a prevalência de osteoporose e fatores clínicos e reprodutivos associados à diminuição da densidade mineral óssea de mulheres climatéricas.

Métodos: realizou-se estudo de corte transversal em que foram avaliados 473 prontuários de mulheres acompanhadas no Ambulatório de Menopausa do CAISM/Unicamp, que estavam em amenorréia há pelo menos 12 meses e tiveram a densidade da massa óssea avaliada por densitometria óssea no Setor de Medicina Nuclear. Foram avaliados: idade, cor, índice de massa corporal, escolaridade, tabagismo, idade à menarca, idade à menopausa, paridade, uso de terapia de reposição hormonal e densidade óssea. A analise estatística foi realizada por meio de regressão logística ajustada por idade e uso de terapia de reposição hormonal. Resultados: a média etária foi de 53,9 anos $(D P \pm 7,1)$ e a média da idade à menopausa, de 45,9 anos (DP $\pm 6,9)$. Na coluna lombar, a prevalência de osteoporose (L2-L4) foi de 14,7\% e de osteopenia, 38\%, ao passo que no fêmur foi de 3,8 e 32,7\%, respectivamente. A regressão logística mostrou que as variáveis associadas à densidade óssea da coluna lombar foram: escolaridade, idade na menarca, idade à menopausa e indice de massa corporal.

Conclusão: a prevalência de osteoporose e osteopenia foi alta. A maior idade, menor escolaridade, menarca tardia, menopausa em idade mais precoce e menor índice de massa corporal foram fatores identificados como de risco para diminuição da massa óssea na população estudada.
\end{abstract}

PALAVRAS-CHAVE: Osteoporose. Menopausa. Densidade mineral óssea.

\section{Introdução}

A osteoporose é enfermidade crônica, multifatorial, muito relacionada ao envelhecimento ${ }^{1}$. Esta doença é de importância crescente, tendo-se em vista o aumento da expectativa de vida populacional, que no Brasil é de aproximadamente 72 anos para as mulheres ${ }^{2}$.

Departamento de Tocoginecologia, Faculdade de Ciências Médicas - CAISM - Unicamp

Correspondência:

Lucia Costa-Paiva

Rua Alexander Fleming, 101 - Cidade Universitária Zeferino $\mathrm{Vaz}$

13083-970 - Campinas - SP

Tel/Fax: (19) 3788-9306 / 3788-9354

e-mail: paivaepaiva@uol.com.br
A maior complicação da osteoporose consiste de fraturas que ocorrem principalmente nas vértebras, punho e colo do fêmur. Na ausência de qualquer procedimento de prevenção ou tratamento, uma em cada duas mulheres aos 70 anos apresentará fraturas de fêmur, e aos 80 anos, duas em três sofrerão o mesmo problema. Constata-se que metade das fraturas de fêmur por osteoporose evoluem para incapacitação parcial ou total. Cerca de 20 a 30\% dos individuos com fratura de colo de fêmur por osteoporose apresentam alterações circulatórias, respiratórias e tromboembólicas, resultando em morte dentro dos dois primeiros anos após a fratura ${ }^{1}$.

Define-se a osteoporose como sendo doença caracterizada por baixa massa óssea e deterioração da microarquitetura do osso, levando a aumen- 
to da fragilidade óssea e, conseqüentemente, maior risco de fraturas ${ }^{3}$. Entretanto, apesar da importância da massa óssea, reunião de consenso recente dos National Health Institutes define a osteoporose como doença caracterizada por resistência óssea comprometida e risco aumentado de fratura ${ }^{4}$.

A identificação precoce das pacientes de risco para osteoporose é de grande importância clínica. Nos últimos anos, vários fatores de risco para osteoporose têm sido identificados.

A idade tem efeito marcante sobre a densidade mineral óssea (DMO). Uma mulher perde aproximadamente metade do seu osso trabecular e $35 \%$ de seu osso cortical durante sua vida. A maior causa desta perda óssea relacionada à idade é provavelmente um decréscimo de formação óssea em nivel celular, resultado da eficiência diminuída dos osteoblastos ${ }^{5}$.

Além da idade, o sexo e a raça estão entre os principais determinantes da massa óssea e do risco de fraturas. Mulheres são mais suscetiveis à osteoporose do que homens, pois além de passarem pela menopausa também possuem menor DMO do que os homens. Fatores genéticos também são responsáveis pelas variações na massa óssea em diferentes grupos éticos e raciais. Indivíduos da raça negra possuem maior pico de massa óssea e, portanto, são menos predispostos a sofrerem de osteoporose que brancos e asiáticos ${ }^{6}$.

$\mathrm{O}$ indice de massa corporal (IMC) está diretamente relacionado à DMO, sendo que os indivíduos obesos têm maior proteção contra osteoporose. Fatores ambientais como hábitos nutricionais, sedentarismo e tabagismo, além de doenças e drogas, também podem estar associados à redução da massa óssea ${ }^{7,8}$.

Em relação aos aspectos reprodutivos, a literatura tem descrito a existência de associação entre as idades na menarca e na menopausa com a extensão do periodo de exposição aos estrogênios, que é um dos mais importantes determinantes da $\mathrm{DMO}^{9}$. Entretanto, a deficiência estrogênica causada pela menopausa é considerada um dos principais fatores de risco para osteoporose em mulheres ${ }^{10}$. Os estrogênios, por sua ação antireabsortiva, atuariam prevenindo a perda de massa óssea, diminuindo o risco de fraturas ${ }^{1,10}$.

Essa prevenção é um dos objetivos da utilização da terapia de reposição hormonal (TRH) em mulheres climatéricas. Estudos recentes têm mostrado que a TRH previne a perda óssea em mulheres na pós-menopausa, diminuindo o risco de fraturas. Entretanto, a TRH em longo prazo não é isenta de riscos e nem todas as pacientes necessitam de reposição ${ }^{11}$. Identificar as mulheres com maior risco deve ser um processo cauteloso, em que o médico deve julgar individualmente cada caso.
Devido à escassez de dados epidemiológicos sobre osteoporose em nossa população e à importância de se identificarem as mulheres de maior risco, realizamos este estudo com o objetivo de conhecer a prevalência da osteoporose e os fatores clínicos e reprodutivos associados à diminuição da DMO de mulheres na pós-menopausa.

\section{Métodos}

Realizou-se estudo de corte transversal com mulheres acompanhadas no Ambulatório de $\mathrm{Me}$ nopausa do CAISM - Unicamp no período de 28 de fevereiro de 2000 a 17 de abril de 2001. Foram revisados 600 prontuários, de acordo com a ordem numérica crescente do registro do Hospital de Clínicas - Unicamp. O tamanho amostral necessário para o estudo foi calculado com base nos fatores de risco para osteoporose de interesse neste estudo. Dentre esses fatores de risco, o IMC foi a variável cujo cálculo resultou em maior tamanho amostral, tendo apresentado prevalência de 16\% de obesas (IMC >30) nas pacientes com osteoporose acompanhadas no Ambulatório de Osteoporose/ Unicamp. Para um erro tipo I $(\mathrm{a}=0,05)$, erro tipo II $(\beta=0,20)$ e uma diferença entre as populações amostral e populacional de 0,15, o tamanho amostral foi calculado em 445 casos $^{12}$.

Foram incluidas no estudo 473 mulheres menopausadas (mínimo de 12 meses de amenorréia) que foram avaliadas por densitometria óssea (Dexa-Lunar DPX, Madison, WI) no Setor de Medicina Nuclear do Hospital das Clínicas da Unicamp. As variáveis independentes estudadas foram: idade, cor, IMC, escolaridade, tabagismo, idade quando da menarca, idade na menopausa, paridade, uso e tempo de uso de TRH. A variável dependente estudada foi a DMO da coluna lombar (L2-L4) e do colo do fêmur, expressa em valores absolutos $\left(\mathrm{g} / \mathrm{cm}^{2}\right.$, gramas de conteúdo mineral ósseo por área ou $\mathrm{cm}^{2}$ de osso analisado) e em valores relativos ao $T$-score (adulto jovem) que corresponde ao número de desvios-padrão acima ou abaixo da média do adulto jovem de referência. Para classificação da DMO foram utilizados os critérios definidos pela Organização Mundial de Saú$\mathrm{de}^{3}$ em valores de $T$-score (adulto jovem) de acordo com as seguintes categorias: normal: valor de DMO dentro de 1 DP da média do adulto jovem de referência; osteopenia: valor de DMO entre - 1 e -2,5 DP da média do adulto jovem de referência, e osteoporose: valor de DMO abaixo de $-2,5 \mathrm{DP}$ da média do adulto jovem de referência.

A análise estatística dos dados foi realizada por meio da elaboração de tabelas descritivas, utilizando-se freqüência, média e desvio padrão. A 
comparação das freqüências foi realizada pelo teste $\chi^{2}$ ou teste exato de Fisher. Realizou-se a análise de regressão logística ajustada por idade e uso de TRH para avaliar quais as variáveis independentes (fatores de risco) que poderiam estar associadas à $\mathrm{DMO}^{13}$. Foi considerado significativo valor de $p<0,05$. Para desenvolvimento destes procedimentos estatísticos foi utilizado o pacote Statistical Analysis System "SAS", versão 6.4.

O protocolo deste estudo foi aprovado pela Comissão de Pesquisa e pelo Comitê de Ética da Faculdade de Ciências Médicas da Unicamp.

\section{Resultados}

A Tabela 1 mostra as características da população estudada. A média etária das mulheres foi de 53,9 anos $( \pm 7,1)$ e a média de idade à menopausa, de 45,9 anos $( \pm 6,9)$. Aproximadamente $64 \%$ delas estavam em uso de TRH e o tempo médio de uso foi de 32,7 meses $( \pm 39,0)$. Cerca de $86 \%$ das mulheres avaliadas eram brancas, de baixa escolaridade e na sua maioria não obesas.

Tabela 1 - Características epidemiológicas e antecedentes clínicos das mulheres estudadas $(n=473)$

\begin{tabular}{lcc}
\hline Característica & Média & Desvio padrão \\
\hline Idade (anos) & 53,9 & 7,13 \\
Menarca (anos) & 13,0 & 1,70 \\
Idade menopausa (anos) & 45,9 & 6,99 \\
Tempo TRH atual (meses) & 32,7 & 39,09 \\
Tempo TRH anterior (meses) & 13,6 & 24,10 \\
Índice de massa corporal & 27,2 & 4,81 \\
Escolaridade (anos) & 3,8 & 3,10 \\
Número de gestações* & 2,6 & 0,62 \\
Número de partos & 2,5 & 0,67 \\
Número de abortos* & 1,6 & 0,76
\end{tabular}

${ }^{*}$ Foram excluídas as pacientes que não tiveram nenhuma gestação

As médias da DMO foram $1,069 \mathrm{~g} / \mathrm{cm}^{2}$ $( \pm 0,177)$ para a coluna lombar e $0,912 \mathrm{~g} / \mathrm{cm}^{2}$ $( \pm 0,151)$ para o colo do fêmur. A prevalência de osteoporose foi de $14,7 \%$ na coluna lombar e 3,8\% no colo do fêmur. Aproximadamente metade das mulheres apresentavam diminuição da massa óssea da coluna lombar (Tabela 2).

A Tabela 3 mostra as características clinicas e sociodemográficas das mulheres estudadas segundo a DMO classificada em categorias de $T$-score. Observou-se que a idade estava diretamente associada à DMO $(\mathrm{p}<0,01)$, ou seja, quanto maior a idade, maior a porcentagem de mulheres com osteopenia ou osteoporose. Cerca de $90 \%$ das
Tabela 2 - Distribuição percentual das mulheres segundo categorias de densidade de massa óssea* na coluna lombar e colo do fêmur $(n=473)$.

\begin{tabular}{lcccc}
\hline T-Score & \multicolumn{2}{c}{ Colo do fêmur } & \multicolumn{2}{c}{ Coluna lombar } \\
& $\mathbf{n}$ & \% & $\mathbf{n}$ & \% \\
\hline Osteoporose & 70 & 14,7 & 18 & 3,8 \\
Osteopenia & 180 & 38,0 & 155 & 32,7 \\
Normal & 223 & 47,1 & 300 & 63,4 \\
Total & 473 & 100 & 473 & 100
\end{tabular}

* DMO segundo T-score adulto jovem

mulheres com osteoporose tinham 50 anos ou mais. A análise estatística ajustada por idade e uso de TRH mostrou que as mulheres com menor escolaridade apresentaram mais osteoporose ou osteopenia $(\mathrm{p}=0,02)$, sendo o odds ratio $(\mathrm{OR}) 7,3$ maior de osteoporose nas mulheres sem instrução quando comparadas às mulheres com maior escolaridade. O IMC também se mostrou inversamente associado à DMO (p<0,01), ou seja, as mulheres com maior IMC apresentaram menos osteopenia ou osteoporose (OR de 0,06 a 0,34). Em relação aos fatores reprodutivos, a idade à menarca também mostrou-se significativamente associada à DMO, sendo que mulheres com menarca mais tardia apresentaram maior porcentagem de osteoporose $(p<0,01)(O R$ de 2,38$)$. A idade à menopausa associou-se significativamente à DMO $(\mathrm{p}<0,01)$ (OR de 2,73 a 5,92) (Tabela 4). Não houve associação da DMO com a cor, número de gestações, número de partos ou abortos, tabagismo, uso ou tempo de uso da TRH.

Dentre as mulheres estudadas, $16(2,8 \%)$ referiram uso de alguma medicação que poderia atuar diminuindo a massa óssea, principalmente corticóides e anticonvulsivantes.

\section{Discussão}

A prevalência de osteoporose e osteopenia na população estudada foi alta. A maior idade, menor escolaridade, menarca tardia, menopausa em idade mais precoce e menor IMC foram fatores identificados como de risco para diminuição da massa óssea na população estudada.

No Brasil, devido às dificuldades que cercam a execução de estudos epidemiológicos em um país de dimensões continentais, de padrões culturais diferentes e do pouco conhecimento do médico brasileiro sobre a doença, não existem dados populacionais sobre a osteoporose. Algumas projeções e levantamentos isolados estimam que a osteoporose acomete cerca de $20 \%$ das mulheres brasileiras com mais de 50 anos $^{1}$. Dados americanos mostram que a proporção de mulheres bran- 
cas com osteoporose identificadas por meio de critérios densitométricos é de aproximadamente 15\% entre 50 e 59 anos, elevando-se para $70 \%$ nas mulheres com mais de 80 anos $^{5}$. Neste estudo a prevalência de osteoporose baseada nos mesmos critérios densitométricos foi de aproximadamente $15 \%$ para o femur e aumentou significativamente com o aumento da idade. Somada à osteopenia, a osteoporose esteve presente em metade das mulheres climatéricas avaliadas. Essas cifras podem ser consideradas altas por se tratar de país tropical e de população relativamente jovem quando comparados a alguns países da Europa e Estados Unidos, porém é necessário considerar que por se tratar de uma população hospitalar, a prevalência pode ter sido superestimada.

Tabela 3 - Características clínicas e sociodemográficas das mulheres segundo categorias de densidade de massa óssea $(T$-score adulto jovem) na coluna lombar ( $=473)$

\begin{tabular}{|c|c|c|c|c|}
\hline & $\begin{array}{c}\text { Osteoporose } \\
\%(n=70)\end{array}$ & $\begin{array}{c}\text { Osteopenia } \\
\%(n=180)\end{array}$ & $\begin{array}{c}\text { Normal } \\
\%(n=223)\end{array}$ & $\begin{array}{l}\text { p valor ajustado * } \\
\text { OR (IC } 95 \%)^{* *}\end{array}$ \\
\hline Idade & & & & $<0,01 \#$ \\
\hline até 39 anos & 1,4 & 3,3 & 4,5 & \\
\hline 40 a 49 anos & 7,2 & 19,4 & 22,4 & \\
\hline 50a 59 anos & 45,7 & 60,6 & 58.3 & \\
\hline 60 anos e mais & 45,7 & 16,7 & 14,8 & \\
\hline Escolaridade & & & & 0,02 \\
\hline sem instrução & 21,4 & 15,6 & 9,4 & $7,34(1,54-34,94)$ \\
\hline $1^{\mathrm{a}}$ a $4^{\mathrm{a}}$ série $/ 1^{\circ} \mathrm{grau}$ & 71,4 & 65,1 & 64,5 & $3,21(0,85-12,15)$ \\
\hline $5^{\mathrm{a}}$ a $8^{\mathrm{a}}$ série $/ 1^{\circ}$ grau & 7,1 & 11,0 & 15,2 & Referência \\
\hline $2^{\circ}$ grau e mais & 0,0 & 8,3 & 10,9 & Referência \\
\hline Cor & & & & 0,18 \\
\hline branca & 81.4 & 89.4 & 85.2 & \\
\hline não branca & 18,6 & 10,6 & 14,8 & \\
\hline Índice de massa corporal & & & & $<0,01$ \\
\hline$<25$ & 50,0 & 30,0 & 22,4 & $0,34(0,18-0,66)$ \\
\hline 25 a 29 & 34,3 & 48,3 & 45,3 & $0,32(0,13-0,74)$ \\
\hline 30 a 34 & 14,3 & 16,1 & 20,6 & $0,06(0,01-0,48)$ \\
\hline 35 e mais & 1,4 & 5,6 & 11,7 & Referência \\
\hline Tabagismo & & & & 0,33 \\
\hline Sim & 15,6 & 14,6 & 11,3 & \\
\hline Não & 84,4 & 85,4 & 88,7 & \\
\hline
\end{tabular}

\#p valor bruto

*Ajustado pela idade e TRH.

${ }^{* *}$ OR (IC 95\%) entre os grupos de osteoporose e normal

Clinicamente, os fatores considerados de maior risco para osteoporose incluem, além da idade, menor IMC, história familiar de osteoporose, estados de deficiência estrogênica e uso de corticosteróides $^{14}$. Nesta casuística foram identificados como fatores significativamente associados à osteoporose, além da idade, o menor IMC e a menor escolaridade. A literatura mostra claramente correlação positiva entre o peso corporal e a massa óssea. Indivíduos com maior IMC apresentam maior DMO. O peso corporal interage com os hormônios gonadais na manutenção da massa óssea, protegendo contra os efeitos adversos da deficiência estrogênica sobre o esqueleto ${ }^{15}$. A associação com a menor escolaridade, nesta população, pode ser indiretamente interpretada como indicativo do estado nutricional porque pode, se em condições inadequadas, limitar o pico e a ma- nutenção da massa óssea.

Quanto aos fatores reprodutivos, foram identificados como associados ao maior risco de osteoporose a menarca tardia e a menopausa em idade mais precoce, que traduzem o tempo de exposição aos estrogênios endógenos. Diversos estudos têm mostrado correlação entre a menarca precoce e altos valores de $\mathrm{DMO}^{9,16,17}$. A idade à menopausa também tem sido positivamente relacionada com a densidade óssea ${ }^{9,18}$.

Chama a atenção o fato de não encontrarmos no presente estudo associação entre a DMO e o uso da TRH. Isso pode ser atribuído ao fato de se tratar de estudo retrospectivo em que os dados referentes ao tipo e tempo de uso da TRH foram sido coletados de informações fornecidas pelas pacientes em consultas de rotina no serviço, ou talvez ao pouco tempo médio de uso. Sem dúvida, essa 
Tabela 4 - Características reprodutivas das mulheres e uso de reposição hormonal segundo categorias de densidade de massa óssea $(T$-score adulto jovem) na coluna lombar ( $\mathrm{N}=473)$.

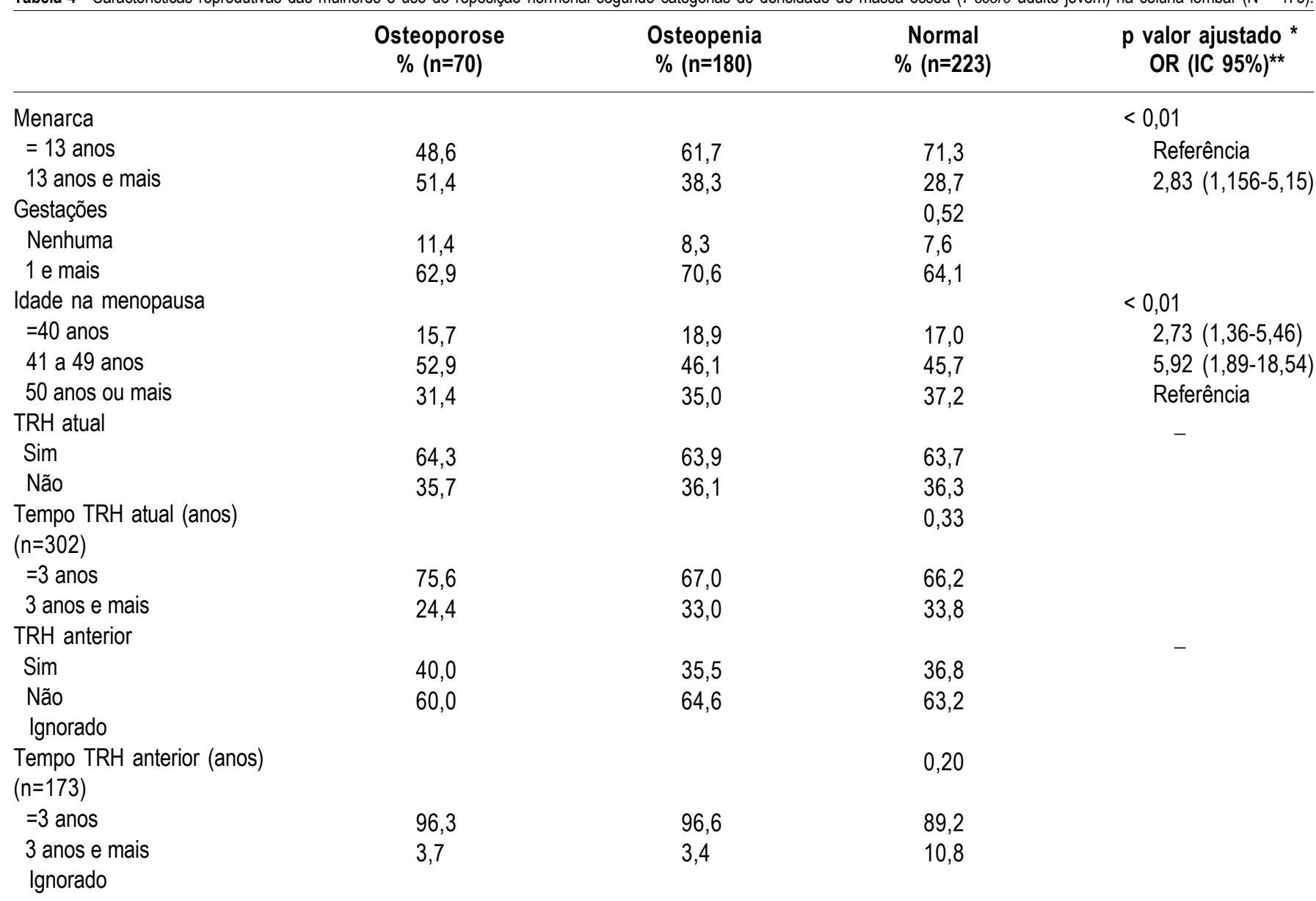

${ }^{*}$ Ajustado pela idade e uso de TRH.

${ }^{\star *}$ OR (IC 95\%) entre os grupos de osteoporose e normal

forma de coleta de dados acrescenta um viés de recordação diferente do que se observa em ensaios clínicos desenhados com o objetivo primário de avaliar os efeitos da intervenção. Muitas evidências na literatura indicam que as doses adequadas de estrogênios administrados em longo prazo podem prevenir a perda de massa óssea em mulheres na pós-menopausa ${ }^{19,20}$. Recentemente os resultados de grande ensaio clínico realizado com mulheres na pós-menopausa que receberam TRH por período de cinco anos mostraram redução de $34 \%$ no risco de fraturas por osteoporose ${ }^{11}$.

É importante ressaltar que o objetivo principal da prevenção da osteoporose é a redução do risco de fraturas. Muitas das diferenças nas taxas de fraturas entre as populações parecem ser explicadas por diferenças sociais e geográficas no pico de massa óssea e na taxa de perda de massa óssea; entretanto, diferenças na geometria óssea, freqüência de quedas e prevalência de outros fatores de risco também têm importante papel. O risco de fratura tem sido consistentemente associado com a historia de quedas, baixa função física, diminuição da força do quadril, diminuição da cognição, visão e presença de armadilhas ambientais ${ }^{4}$. Fatores associados com aumento no risco de quedas, como diminuição sensorial, enfraquecimento muscular e instabilidade, também precisam ser identificados.

Alguns autores consideram, entretanto, que os fatores de risco têm, mesmo em combinação, baixa sensibilidade e especificidade para predizer baixa densidade óssea ${ }^{21,22}$. Entretanto, esses fatores podem ser usados para ajudar a identificar as mulheres de alto risco que poderiam se beneficiar de avaliações adicionais ${ }^{14,21}$.

O conhecimento dos fatores de risco para baixa massa óssea identificados neste estudo poderá auxiliar os profissionais na identificação de pacientes de risco para osteoporose, uma vez que as evidências demonstram que a associação de dados da história clínica, exame físico e de exames complementares específicos é essencial para a prevenção das fraturas osteoporóticas. Outros estudos com dados nacionais, particularmente estudos prospectivos populacionais, são necessários para maior elucidação sobre prevalência e fatores de risco para osteoporose em nossa população. 


\section{ABSTRACT}

Purpose: to evaluate the prevalence of osteoporosis in climacteric women and analyze the influence of general and reproductive risk factors on bone mineral density.

Methods: a cross-sectional study with the evaluation of the 473 hospital records of climacteric women followed up at the Menopause Outpatient Facility of CAISM/Unicamp, between 03/28/2000 and 04/17/2001. These women were at least 12 months in amenorrhea and presented the results of a bone densitometry study performed at the Nuclear Medicine Department of HC/Unicamp. The following variables were evaluated: age, color, body mass index, level of education, smoking, use of medication, age at menopause, parity, use and length of hormone replacement therapy and its effect on bone mineral density. Statistical analyses were performed using logistic regression ajusted by age and hormone replacement therapy use.

Results: the mean age of the studied women was 53.9 years ( \pm 7.1 SD) with mean age at menopause being 45.9 years ( \pm 6.9 SD). Osteoporosis occurred in $14.7 \%$ and osteopenia in $38 \%$ of the cases in the lumbar vertebrae ( $L_{2}-L_{4}$ interspace) and in 3.8 and $32.7 \%$ in the femur, respectively. Logistic regression adjusted to age and hormone therapy showed an association between the following variables: level of education, age at menopause and body mass index.

Conclusion: there was a high prevalence of osteoporosis and osteopenia in the studied population. Advanced age, lower level of education, late menarche, early menopause and lower body mass index were identified as risk factors for developing decreased bone mass in the studied population.

KEYWORDS: Menopause. Osteoporosis. Bone mineral density. Risk factors.

\section{Referências}

1. National Consensus Proposal. Osteoporosis 1995 basic diagnosis and therapeutic elements. São Paulo Med J 1995; 113 Suppl:7-18.

2. DATASUS: banco de dados. [on line] 2003. Disponível em: http://www.datasus.gov.br/

3. World Health Organization. Assessment of fracture risk and its application to screening for postmenopausal osteoporosis. Geneva: WHO; 1994. (Technical Reports Series).

4. NIH Consensus Development Panel on Osteoporosis Prevention, Diagnosis and Therapy. Osteoporosis prevention, diagnosis and therapy. JAMA 2001; 285:785-95.

5. Riggs BL, Melton LJ. Involutional osteoporosis. N Engl J Med 1986; 314:676-86.

6. Radominski SC, Pinto-Neto AM, Marinho RM, et al. Osteoporose pós-menopausa: Diretrizes da Associação Médica Brasileira e Conselho Federal de Medicina. [on line] 2002. Disponivel em: http:// www.org.br/.
7. Holm K, Dan A, Wilbur J, Li S, Walker J. A longitudinal study of bone density in midlife women. Health Care Women Int 2002; 23:678-91.

8. Brown JP, Josse RG. 2002 clinical practice guidelines for the diagnosis and management of osteoporosis in Canada. CMAJ 2002; 167 Suppl:S1-34.

9. Ito M, Yamada M, Hayashi K, Ohki M, Uetani M, Nakamura T. Relation of early menarche to high bone mineral density. Calcif Tissue Int 1995; 57:11-4.

10.Szejnfeld VL. Alterações ósseas: fisiopatologia, diagnóstico e tratamento. In: Fernandes $\mathrm{CE}$, editor. Menopausa: diagnóstico e tratamento. $1^{\text {a }}$ ed. São Paulo: Serguimento; 2003. p.49-9.

11.Roussow JE, Anderson GL, Prentice RL, et al. Risks and benefits of estrogen plus progestin in health postmenopausal women: principal results from the Women's Health Initiative randomized controlled trial. JAMA 2002; 288:321-33.

12.Snedecor GW, Cochran WG. Statistical methods. $7^{\text {th }}$ ed. Ames: Iowa State University Press; 1980.

13.Draper NR, Smith H. Applied regression analysis. $2^{\text {nd }}$ ed. New York: John Wiley \& Sons; 1981.

14.Cummings SR, Nevitt MC, Browner WS, et al. Risk factors for hip fracture in white women. Study of Osteoporotic Fractures Research Group. N Engl J Med 1995, 332:767-73.

15.Heiss CJ, Sanborn CF, Nichols DL, Bonnick SL, Alford BB. Associations of body fat distribution, circulating sex hormones, and bone density in postmenopausal women. J Clin Endocrinol Metab 1995; 80:1591-6.

16. Cooper GS, Sandler DP. Long-term effects of reproductive-age menstrual cycle patterns on periand postmenopausal fracture risk. Am J Epidemiol $1997 ; 145: 804-9$

17.Osey-Hyiaman D, Satoshi T, Ueji M, Hideto T, Kano $\mathrm{K}$. Timing of menopause, reproductive years, and bone mineral density: a cross-sectional study of postmenopausal Japanese women. Am J Epidemiol 1998; 148:1055-61.

18. O’Neill TW, Silman AJ, Naves-Diaz M, Cooper C, Kanis J, Felsenberg D. Influence of hormonal and reproductive factors on the risk of vertebral deformity in European women. Osteoporos Int 1997; 7:72-8.

19.Lindsay R. The menopause sex steroids and osteoporosis. Clin Obstet Gynecol 1987; 30:847-59.

20.Torgerson DJ, Bell-Syer SE. Hormone replacement therapy and prevention of nonvertebral fractures: a meta-analysis of randomized trials. JAMA 2001; 285:2891- 97.

21.Lydick E, Zimmerman SI, Yawn B, et al. Development and validation of a discriminative quality of life questionnaire for osteoporosis. J Bone Miner Res 1997; 12:456-63.

22. Cadarette SM, Jaglal SB, Murray TM, et al. Evaluation of decisions rules for referring women for bone densitometry by dual energy $\mathrm{x}$-ray absorptiometry. JAMA 2001; 286:57-63.

Recebido em: 18/8/2003 Aceito com modificações em: 30/8/2003 